\title{
MIMO Self-Encoded Spread Spectrum with Iterative Detection over Rayleigh Fading Channels
}

\author{
Shichuan Ma, Lim Nguyen, Won Mee Jang, and Yaoqing (Lamar) Yang \\ Department of Computer and Electronics Engineering, University of Nebraska-Lincoln, Omaha, NE 68182, USA \\ Correspondence should be addressed to Shichuan Ma, sma@huskers.unl.edu
}

Received 15 February 2010; Revised 15 July 2010; Accepted 16 August 2010

Academic Editor: Christian Schlegel

Copyright () 2010 Shichuan Ma et al. This is an open access article distributed under the Creative Commons Attribution License, which permits unrestricted use, distribution, and reproduction in any medium, provided the original work is properly cited.

Self-encoded spread spectrum (SESS) is a novel communication technique that derives its spreading code from the randomness of the source stream rather than using conventional pseudorandom noise (PN) code. In this paper, we propose to incorporate SESS in multiple-input multiple-output (MIMO) systems as a means to combat against fading effects in wireless channels. Orthogonal space-time block-coded MIMO technique is employed to achieve spatial diversity, and the inherent temporal diversity in SESS modulation is exploited with iterative detection. Simulation results demonstrate that MIMO-SESS can effectively mitigate the channel fading effect such that the system can achieve a bit error rate of $10^{-4}$ with very low signal-to-noise ratio, from $3.3 \mathrm{~dB}$ for a $2 \times 2$ antenna configuration to just less than $0 \mathrm{~dB}$ for a $4 \times 2$ configuration under Rayleigh fading. The performance improvement for the $2 \times 2$ case is as much as $6.7 \mathrm{~dB}$ when compared to an MIMO PN-coded spread spectrum system.

\section{Introduction}

Self-encoded spread spectrum (SESS) and multiple access communications have been proposed and shown to have a number of unique features $[1,2]$. By deriving its spreading sequences from the randomness of the source stream, SESS provides a feasible implementation of random-coded spread spectrum and potentially enhances the transmission security. Since the use of PN code generators is obviated, SESS can simplify multirate transmissions with variable processing gains in multimedia applications [3]. In previous works, we have shown that the modulation memory in SESS can yield signal gain in AWGN channels [4]. We have also demonstrated that the inherent temporal diversity in SESS modulation can be exploited with iterative detection to significantly improve the system performance over timevarying fading channels [5].

It is also well known that $\mathrm{PN}$-coded spread spectrum can be incorporated with multiple-input multiple-output (MIMO) techniques to achieve multipath and spatial diversities [6-8]. In delay-spread or frequency-selective fading, the system performance can be improved with a Rake receiver that can resolve and combine the multi-path signal components by matching the Rake fingers with the delayed PN codes to achieve multi-path diversity. By deploying multiple antennas at both transmitter and receiver, MIMO architectures are capable of mitigating channel fading by taking advantage of the spatial diversity and enhancing system capacity by employing spatial multiplexing [9-11] .

In this paper, we propose a novel approach to combat against fading in wireless channels by incorporating SESS in MIMO system. Our motivation is based on the observation that SESS not only can achieve multi-path diversity like PNcoded spread spectrum, but it can also provide both inherent temporal diversity and signal gain. Our work combines orthogonal space-time block-coded MIMO technique and iterative detection to obtain spatial and temporal diversities, respectively. We determine the bit error rate (BER) performance of MIMO-SESS system over Rayleigh fading channels and show that the fading effects can be completely mitigated by exploiting diversities in both space and time domains. The proposed scheme in this paper employs only one spreading sequence which we refer to as the single-code scheme. Recently, we have reported a multicode Alamouti scheme that can enhance the system throughput [12] with multiple spreading sequences, but at the expense of a BER 


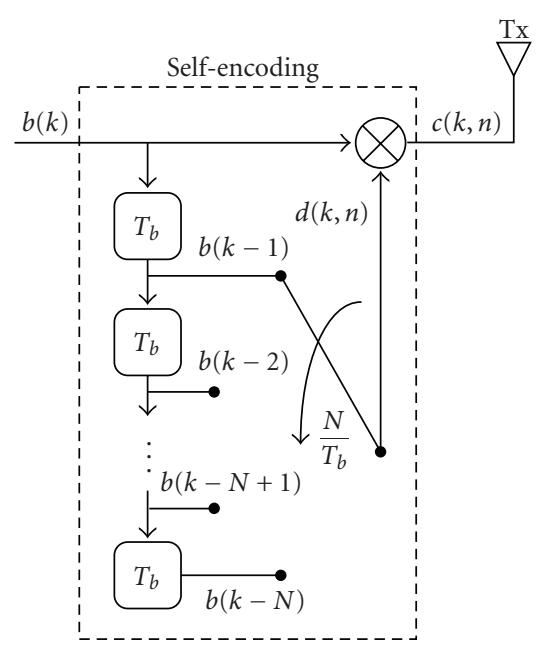

(a) Transmitter

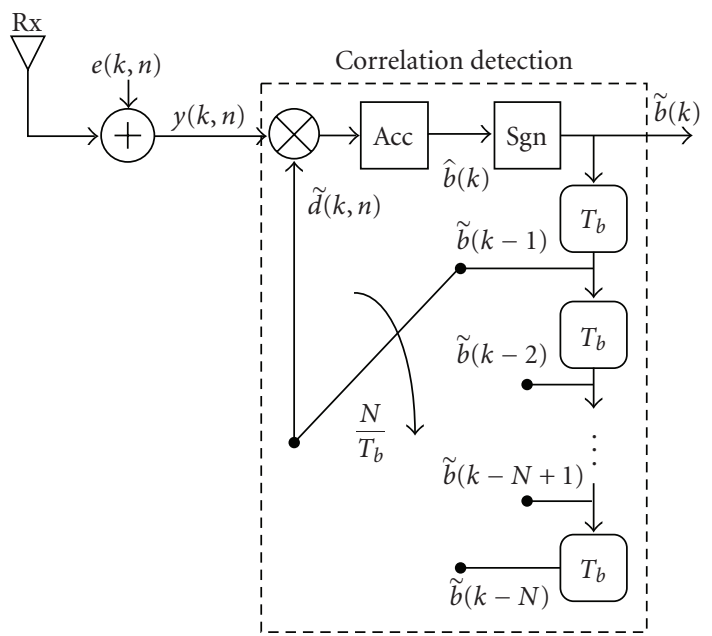

(b) Receiver

FIgURE 1: Block diagram of SESS system.

degradation. The orthogonal space-time block codes and multiple iterations in this paper thus could be incorporated into the multi-code scheme in order to improve the BER performance.

The rest of this paper is organized as follows. In Section 2, we briefly describe the original SESS system. The proposed MIMO-SESS with iterative detection is described in Section 3. Section 4 presents the simulation results and compares the proposed system to an MIMO PN-coded spread spectrum (MIMO-PNSS) system. Finally, Section 5 concludes this paper.

Notations. The superscripts $*$ and $T$ represent the conjugate and the transpose operations, respectively. $|\cdot|, E\{\cdot\}$, and $\operatorname{diag}(\cdot)$ denote the absolute value of a scalar, the expectation operation, and the diagonal vector of a matrix, respectively. Matrix (vector) is represented by capital (small) bold letter, with $a(k, n)$ representing the $n$th element of vector $\mathbf{a}(k)$.

\section{Background}

In this section, we briefly review the concept of self-encoding spread spectrum. The readers are referred to the original description in [1] for more details.

2.1. Transmitter. A block diagram of SESS system is shown in Figure 1, where each rounded corner block represents one delay register, $N$ denotes the length of spreading sequence, and $T_{b}$ is the bit duration.

The source information $b$ is assumed to be bipolar values of \pm 1 . The bits are first spread by the self-encoded spreading sequence $\mathbf{d}(k)$ of length $N$ at a chip rate of $N / T_{b}$. This sequence is constructed from the source information stored in the delay registers that are updated every $T_{b}$. For example, the spreading sequence for the $k$ th bit, $b(k)$, is given as

$$
\mathbf{d}(k)=[b(k-1) b(k-2) \cdots b(k-N)]^{T} .
$$

Thus, with a random input data stream, the sequence is also random and time varying from one bit to another. We assume that the delay registers have been seeded by a randomly selected sequence (which has also been acquired at the receiver to initialize the despreading process).

The spread spectrum chips are then transmitted as

$$
\mathbf{c}(k)=b(k) \mathbf{d}(k) \text {. }
$$

Clearly, SESS signal has a modulation memory depth equal to $N$ :

$$
c(k, n)=b(k) d(k, n)=b(k) b(k-n) .
$$

2.2. Receiver. We assume that the chips are transmitted over a wireless channel and each bit experiences independent Rayleigh fading that is constant over the bit duration. The channel coefficient for the $k$ th bit interval is represented by $\alpha(k)$. The received signal $y(k, n)$ can be expressed as

$$
y(k, n)=\alpha(k) c(k, n)+e(k, n),
$$

where the additive Gaussian noise $e(k, n)$ has zero mean and variance $N N_{0} / 2$. Note that the noise is sampled at the chip rate and is broadband; its variance is thus the narrow-band noise variance $N_{0} / 2$ multiplied by the spreading factor $N$. from

The soft correlation estimate of the $k$ th bit is computed

$$
\begin{aligned}
\hat{b}(k) & =\frac{1}{N} \sum_{n=1}^{N} y(k, n) \tilde{d}(k, n) \\
& =\frac{1}{N} \sum_{n=1}^{N} \alpha(k) c(k, n) \tilde{d}(k, n)+u(k),
\end{aligned}
$$

where

$$
u(k)=\frac{1}{N} \sum_{n=1}^{N} e(k, n) \tilde{d}(k, n)
$$


is the narrow-band Gaussian noise component of the correlation output and $\tilde{d}(k, n)$ is the $n$th chip of the despreading sequence $\widetilde{\mathbf{d}}(k)$ generated by the delay registers. We assume that the receiver delay registers have been initialized by the same seed sequence at the transmitter. The content of the delay registers, $\tilde{\mathbf{d}}(k)$, are then updated by the hard decision of the correlation estimate, $\widetilde{b}(k)=\operatorname{sgn}[\hat{b}(k)]$. Since the hard decision may be erroneous, the reconstructed despreading sequence could be different from the self-encoded spreading sequence at the transmitter. A bit error therefore will propagate through the delay registers for the next $N$ bits and cause self-interference that attenuates the strength of the despread signal $\hat{b}(k)$. The correlation estimate given in (5) can be written as

$$
\hat{b}(k)=\frac{1}{N} \sum_{n=1}^{N} \alpha(k) c(k, n) \tilde{b}(k-n)+u(k) .
$$

Substituting (3) into (7), we have

$$
\hat{b}(k)=\alpha(k)\left[\frac{1}{N} \sum_{n=1}^{N} b(k-n) \tilde{b}(k-n)\right] b(k)+u(k) .
$$

Thus, the hard decision is performed on the soft estimate from the correlator output, a noisy and fading signal that is also subjected to self interference (expressed by the term inside the square bracket in (8)):

$$
\tilde{b}(k)=\operatorname{sgn}[\hat{b}(k)]= \begin{cases}1, & \hat{b}(k)>0, \\ -1, & \hat{b}(k)<0 .\end{cases}
$$

\section{MIMO-SESS with Iterative Detection}

In this section, we seek to improve the performance of SESS system in fading channels by employing multiple antennas to take advantage of spatial diversity and by utilizing iterative detection to achieve temporal diversity. Figure 2 shows a block diagram of the proposed MIMO-SESS system.

At the transmitter, the information bits first undergo spread spectrum modulation according to the self encoding procedure in Section 2.1. The spread spectrum chips are further encoded using orthogonal space-time block codes (OSTBCs) and then transmitted over an $N_{t} \times N_{r}$ MIMO fading channel, where $N_{t}$ antennas and $N_{r}$ antennas are deployed at the transmitter and receiver, respectively. We assume that the channel between each transmit/receive antenna pair is subjected to Rayleigh fading that remains constant over $T_{b}$ but is independent from bit to bit [5, 13]. Furthermore, the channels for different transmit/receive antenna pairs experience independent fading. The signals from the received antennas are combined linearly to generate the output that is then detected with an iterative algorithm for bit recovery.

3.1. MIMO Transmission. In the above MIMO-SESS system, the (inner) MIMO encoding is independent from (outer) self-encoding as can be seen from Figure 2. The decoupling nature of this approach simplifies decoding at the receiver and lets us employ, in principle, any MIMO techniques for signal transmission, such as beamforming for spatial filtering [14] and for signal quality improvement [15-17], space-time block coding for spatial diversity [18-20], and layered space-time architecture for spatial multiplexing [2123]. In this paper, we adopt OSTBC which provides full diversity gain and allows linear maximum-likelihood (ML) decoding [24].

Numerous OSTBCs have been reported in the literature. In this work, we employ the full-rate code $g_{2}$ for the twotransmit-antenna case, the half-rate code $g_{3}$ for the threetransmit-antenna case, and the half-rate code $g_{4}$ for the fourtransmit-antenna case. The full-rate code $g_{2}$ was proposed in [25] and is known as the Alamouti scheme. The half-rate codes $g_{3}$ and $g_{4}$ were developed in [24]. The code matrices have been reproduced as follows:

$$
\begin{gathered}
g_{2}=\left(\begin{array}{cc}
s_{1} & s_{2} \\
-s_{2}^{*} & s_{1}^{*}
\end{array}\right), \\
g_{3}=\left(\begin{array}{ccc}
s_{1} & s_{2} & s_{3} \\
-s_{2} & s_{1} & -s_{4} \\
-s_{3} & s_{4} & s_{1} \\
-s_{4} & -s_{3} & s_{2} \\
s_{1}^{*} & s_{2}^{*} & s_{3}^{*} \\
-s_{2}^{*} & s_{1}^{*} & -s_{4}^{*} \\
-s_{3}^{*} & s_{4}^{*} & s_{1}^{*} \\
-s_{4}^{*} & -s_{3}^{*} & s_{2}^{*}
\end{array}\right), \\
g_{4}=\left(\begin{array}{cccc}
s_{1} & s_{2} & s_{3} & s_{4} \\
-s_{2} & s_{1} & -s_{4} & s_{3} \\
-s_{3} & s_{4} & s_{1} & -s_{2} \\
-s_{4} & -s_{3} & s_{2} & s_{1} \\
s_{1}^{*} & s_{2}^{*} & s_{3}^{*} & s_{4}^{*} \\
-s_{2}^{*} & s_{1}^{*} & -s_{4}^{*} & s_{3}^{*} \\
-s_{3}^{*} & s_{4}^{*} & s_{1}^{*} & -s_{2}^{*} \\
-s_{4}^{*} & -s_{3}^{*} & s_{2}^{*} & s_{1}^{*}
\end{array}\right) .
\end{gathered}
$$

In (10), (11), and (12), each row includes the symbols to be transmitted in one time slot via $N_{t}$ transmit antennas, and each column consists of the symbols in one block for each antenna. The block sizes here are 2, 8, and 8 for the two-, three-, and four-antenna cases, respectively.

In order to enable linear maximum likelihood decoding of OSTBC, the channel should be stable in one block. Since we have assumed that the channel fading remains constant over a bit duration, this implies that the spreading factor $N$ should be a multiple of the block size. Notice that the total transmit power of MIMO system should be the same as single-antenna system. This means that the equivalent MIMO power per transmit antenna must be normalized by $N_{t}$. 


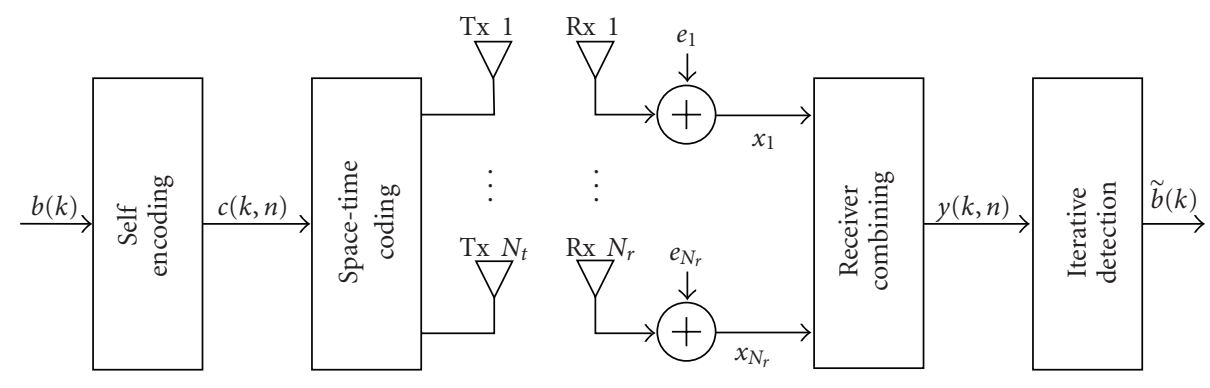

(a) Transmitter

(b) Receiver

FIGURE 2: Block diagram of the proposed MIMO-SESS system.

3.2. Space-Time Processing Example. In the following, we will use the Alamouti scheme for two transmit antennas as an example to describe space-time block coding and decoding procedures.

At the transmitter, the spread spectrum chips are divided into two streams according to $g_{2}$. A block of two consecutive chips, $c(k, 2 i)$ and $c(k, 2 i+1)$, are transmitted by sending $c(k, 2 i)$ and $-c^{*}(k, 2 i+1)$ to the first antenna, and $c(k, 2 i+1)$ and $c^{*}(k, 2 i)$ to the second antenna. Here $i \in\{0,1, \ldots, N / 2-$ $1\}$ is the block index for the chips of the $k$ th bit. We note that in this case the power per antenna is one-half of the total power. The signals are then transmitted over an MIMO fading channel.

At the receiver, the signals from the antennas are first combined to achieve spatial diversity. For the $m$ th receive antenna, the signals within the $i$ th block are given as

$$
\begin{aligned}
x_{m}(k, 2 i)= & h_{1, m}(k) c(k, 2 i) \\
& +h_{2, m}(k) c(k, 2 i+1)+e_{m}(k, 2 i), \\
x_{m}(k, 2 i+1)= & -h_{1, m}(k) c^{*}(k, 2 i+1) \\
& +h_{2, m}(k) c^{*}(k, 2 i)+e_{m}(k, 2 i+1),
\end{aligned}
$$

where $h_{1, m}(k)$ and $h_{2, m}(k)$ denote the complex channel impulse response coefficients (for the $k$ th bit) between the $m$ th receive antenna and the first-and second-transmit antennas, respectively. $e_{m}$ is the Gaussian noise with zero mean and variance $N N_{0} / 2$.

Again we assume that the delay registers in the receiver have been initially synchronized with the transmitter [26], and that perfect channel knowledge is available. Under these assumptions, diversity combining is carried out over two consecutive chip intervals per $[11,25]$

$$
\begin{aligned}
y(k, 2 i)= & \sum_{m=1}^{N_{r}}\left(h_{1, m}^{*}(k) x_{m}(k, 2 i)\right. \\
& \left.\quad+h_{2, m}(k) x_{m}^{*}(k, 2 i+1)\right)+w(k, 2 i) \\
= & \alpha(k) c(k, 2 i)+w(k, 2 i),
\end{aligned}
$$

$$
\begin{aligned}
y(k, 2 i+1)= & \sum_{m=1}^{N_{r}}\left(h_{2, m}^{*}(k) x_{m}(k, 2 i)\right. \\
& \left.\quad-h_{1, m}(k) x_{m}^{*}(k, 2 i+1)\right)+w(k, 2 i+1) \\
= & \alpha(k) c(k, 2 \mathrm{i}+1)+w(k, 2 i+1),
\end{aligned}
$$

where

$$
\begin{gathered}
\alpha(k)=\sum_{m=1}^{N_{r}}\left(\left|h_{1, m}(k)\right|^{2}+\left|h_{2, m}(k)\right|^{2}\right), \\
w(k, n)=\left\{\begin{array}{rr}
\sum_{m=1}^{N_{r}}\left(h_{1, m}^{*}(k) e_{m}(k, n)+h_{2, m}(k) e_{m}^{*}(k, n+1)\right), \\
\text { if } n \text { is even, } \\
\sum_{m=1}^{N_{r}}\left(h_{2, m}^{*}(k) e_{m}(k, n-1)-h_{1, m}(k) e_{m}^{*}(k, n)\right), \\
\text { if } n \text { is odd }
\end{array}\right.
\end{gathered}
$$

The combined signals given in (15) and (16) can be expressed as

$$
y(k, n)=\alpha(k) c(k, n)+w(k, n)
$$

and further in vector form

$$
\mathbf{y}(k)=\alpha(k) \mathbf{c}(k)+\mathbf{w}(k) .
$$

Similar signal models and analysis can be developed for the three- and four-transmit-antenna cases. In general for an $N_{t} \times N_{r}$ MIMO system, (19) and (20) remain valid with

$$
\alpha(k)=\sum_{n=1}^{N_{t}} \sum_{m=1}^{N_{r}}\left|h_{n, m}(k)\right|^{2},
$$

albeit with a more complicated noise term $\mathbf{w}(k)$.

3.3. Iterative Detection. Since SESS signal has modulation memory, its optimum detection is the maximum likelihood sequence detector or the Viterbi detector. However, the complexity of the optimum detection grows exponentially as 


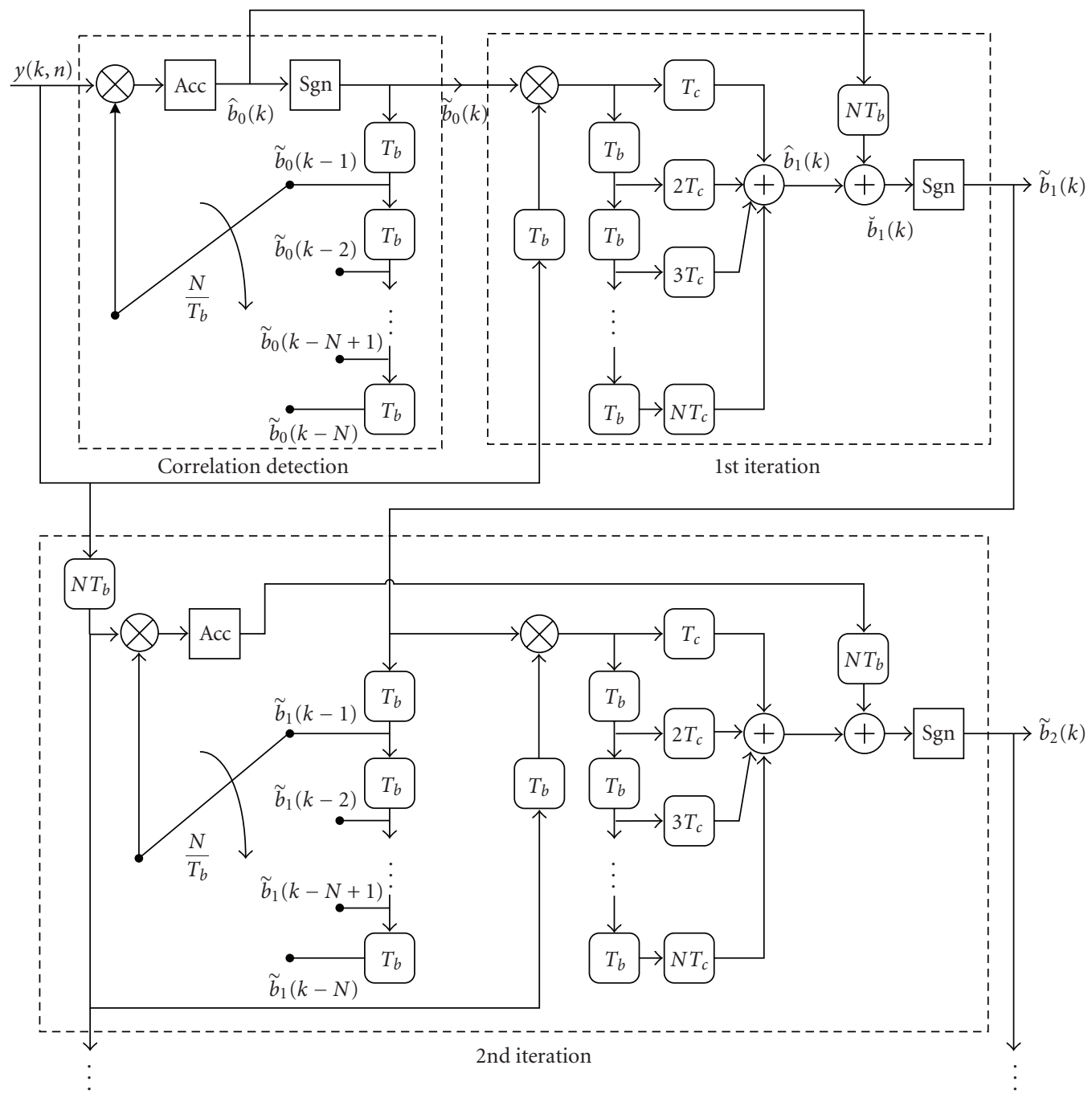

FIGURE 3: Block diagram of iterative detection.

$2^{N}$, making it impractical to implement unless $N$ is rather small. On the other hand, iterative detection is suboptimum but linear in $N$, and can converge iteratively toward the optimum performance.

The iterative detection for SESS is described by the detailed block diagram illustrated in Figure 3 . Notice that correlation detection provides the initial estimate $\tilde{b}_{0}(k)$ for the iterative detection. For simplicity we will ignore self interference in the following signal analysis, so that $\sum_{n=1}^{N} b(k-n) \tilde{b}(k-n)=N$. This in fact is justified if the BER is sufficiently low. According to the description in Section 2, the soft correlation output or estimate of the $k$ th bit is then given as

$$
\widehat{b}_{0}(k)=\alpha(k) b(k)+v_{1}(k)
$$

where $v_{1}(k)$ is the noise term.

The hard decision of the correlation output $\widetilde{b}_{0}(k)=$ $\operatorname{sgn}\left[\hat{b}_{0}(k)\right]$ is then used to construct the despreading sequences.

Now assuming that we have obtained the correlation detection of $b(k)$, then based on (3) and (19) the next $N^{2}$ chips of bits $b(k+1), b(k+2), \ldots, b(k+N)$ can be written in a square matrix $\mathbf{P}(k)$ as shown by

$$
\mathbf{P}(k)=\left[\begin{array}{cccc}
\alpha(k+1) b(k+1) b(k) & \alpha(k+1) b(k+1) b(k-1) & \cdots & \alpha(k+1) b(k+1) b(k-N+1) \\
\alpha(k+2) b(k+2) b(k+1) & \alpha(k+2) b(k+2) b(k) & \cdots & \alpha(k+2) b(k+2) b(k-N) \\
\vdots & \vdots & \ddots & \vdots \\
\alpha(k+N) b(k+N) b(k+N-1) & \alpha(k+N) b(k+N) b(k+N-2) & \cdots & \alpha(k+N) b(k+N) b(k)
\end{array}\right] .
$$


Each element of this matrix represents a chip as given in (19) and the $j$ th row includes the chips of bit $b(k+j)$. Note that the noise term has been omitted due to the page limitation.

Observe that the $k$ th bit, $b(k)$, are also present in the diagonal elements of the matrix $\mathbf{P}(k)$. This means that the information of the $k$ th bit is also included in the next $N$ bits. Thus, the modulation memory in SESS signals could be utilized to enhance the detection of the $k$ th bit. The iterative detection employs the hard decisions of these next $N$ bits, $\left[\begin{array}{llll}\tilde{b}_{0}(k+1) & \tilde{b}_{0}(k+2) & \cdots & \tilde{b}_{0}(k+N)\end{array}\right]$, in order to accumulate the time-diversity soft estimate of $b(k)$ as

$$
\begin{aligned}
\hat{b}_{1}(k)= & \frac{1}{N} \operatorname{diag}(\mathbf{P}(k))\left[\tilde{b}_{0}(k+1) \tilde{b}_{0}(k+2) \cdots \tilde{b}_{0}(k+N)\right]^{T} \\
& +v_{2}(k) \\
= & \frac{1}{N} \sum_{n=1}^{N} \alpha(k+n) b(k)+v_{2}(k) .
\end{aligned}
$$

where $v_{2}(k)$ is a noise term.

The summation of the correlation estimate, $\hat{b}_{0}(k)$, delayed by $N T_{b}$, and the time-diversity estimate, $\hat{b}_{1}(k)$, provides the soft estimate for the hard decision of the first iteration. That is,

$$
\begin{aligned}
\breve{b}_{1}(k) & =\hat{b}_{0}(k)+\hat{b}_{1}(k) \\
& =\left(\alpha(k)+\frac{1}{N} \sum_{n=1}^{N} \alpha(k+n)\right) b(k)+v(k),
\end{aligned}
$$

where $v(k)=v_{1}(k)+v_{2}(k)$.

The output of the first iteration $\tilde{b}_{1}(k)$ is then obtained by the hard decision of $\breve{b}_{1}(k)$.

This first iterative bit decision $\tilde{b}_{1}(k)$ is an improvement over the initial estimate $\tilde{b}_{0}(k)$ because it is based on the combined soft estimates which yield not only a signal (to noise) gain of $3 \mathrm{~dB}$ but also the time diversity gain from SESS modulation memory. The improved bit decision can be further fed back again to reconstruct the de-spreading sequences for the correlation detection and also to reaccumulate the soft bit estimate from temporal diversity combining in the manner described by (24).

The block diagram for this second iteration is illustrated in the lower part in Figure 3. It is clear from the block diagram that procedure can be repeated iteratively. We note in addition that each iteration introduces a time delay of $N T_{b}$. Furthermore, the detector structure is partly similar to a Rake receiver that employs $N$ fingers to exploit the $N$-fold temporal diversity in SESS signals.

\section{Simulation Results}

In this section, the performance of the proposed MIMOSESS system has been determined by simulations and is compared with a conventional MIMO-PNSS system. The

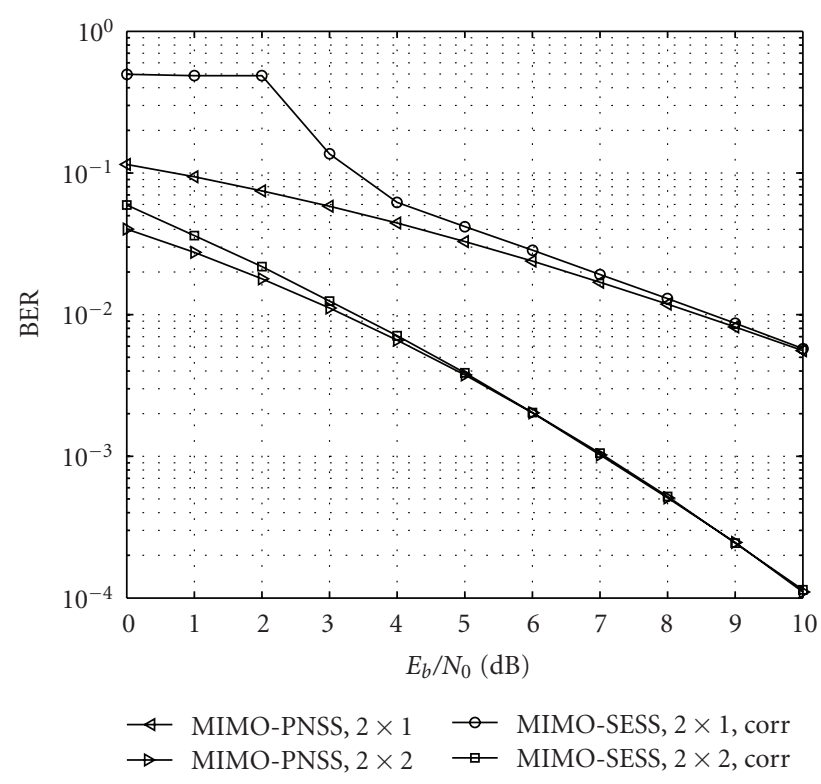

FIgure 4: Comparison between an MIMO-PNSS system and the proposed MIMO-SESS system without iterative detection, two transmit antennas using full-rate code $g_{2}, N=64$.

length of the spreading sequence is set to $N=64$ unless noted otherwise. For each scenario and bit signal-to-noise ratio $E_{b} / N_{o}$ (SNR), 100 runs of 100,000 bits have been simulated to obtain the average bit error rate.

Figure 4 compares two-transmit-antenna MIMO-SESS system without iterative detection to MIMO-PNSS system. Because iterative detection is not applied in this scenario, no temporal diversity gain is achieved. This means that the same performance can be expected with or without self-encoding. However, the MIMO-SESS system degrades at low SNR due to the effects of error propagation [1]. By employing MIMO technique, error propagation can be efficiently alleviated, as shown by the $2 \times 2$ scenario.

The results for the two-transmit-antenna MIMO-SESS system with iterative detection are plotted in Figure 5, which clearly shows that the BER performance has been significantly improved with iterative detection. With only one iteration, there is about $6.7 \mathrm{~dB}$ gain for the $2 \times 2$ scenario at $10^{-4} \mathrm{BER}$. This performance improvement can be attributed to the temporal diversity introduced by selfencoding, in addition to the signal gain from combining the soft estimates. Moreover, the improvement with the second iteration over the first iteration is quite marginal (less than $0.1 \mathrm{~dB}$ at $\left.10^{-4} \mathrm{BER}\right)$. This demonstrates that the algorithm is very efficient and converges extremely fast (to within one iteration in this case). Thus, in practice, the second iteration may not be necessary nor desirable given the diminishingly small gain and the increased computational complexity and delays.

Figures 7 and 6 show the performance of the MIMOSESS system with three- and four-transmit-antenna configurations, respectively. Clearly, the spatial diversity gains have increased compared to the two-transmit-antenna case 


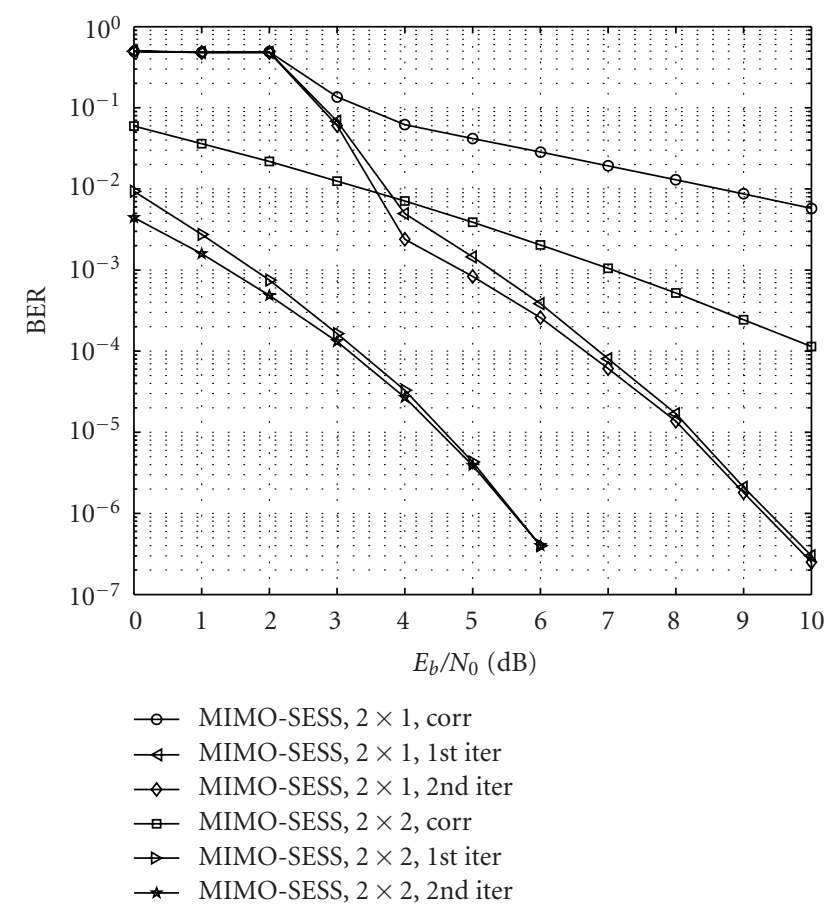

FIgure 5: Performance of the proposed MIMO-SESS system with iterative detection, two transmit antennas using full-rate code $g_{2}, N$ $=64$.

as evident from the steeper slopes of the BER curves. This can also be quantified from the plots by observing, for example, that the overall gain at $10^{-4}$ BER has decreased from $6.7 \mathrm{~dB}$ for the $2 \times 2$ scenario to about $5.3 \mathrm{~dB}$ and $4.5 \mathrm{~dB}$ for the $3 \times 2$ and $4 \times 2$ configurations, respectively. Again, the performance gain with the iterative detection is significant for all antenna configurations. In particular, the $4 \times 2$ configuration requires an $E_{b} / N_{o}$ of just less than $0 \mathrm{~dB}$ at $10^{-4}$ BER. It should be noted that these results also reflect additional coding gains (compared to the results in Figure 5) since the space-time codes are half rates.

Finally, Figure 8 shows the effects of varying the spreading lengths on the example $2 \times 2$ MIMO-SESS system. The performance of a BPSK system under AWGN and a $2 \times 2$ MIMO-PNSS system under Rayleigh fading has also been plotted for comparison. The plots show that the performance improves with the spreading length (especially at low SNR) because the temporal diversity increases with $N$ (more Rake fingers) as would be expected. When compared to the BPSK system under AWGN, the performance gain is nearly $5 \mathrm{~dB}$ at $10^{-4} \mathrm{BER}$, demonstrating that the fading effect on the BER has been completely mitigated with MIMO-SESS. The results also show that this performance can be achieved with a relatively small value of $N=64$.

\section{Conclusion}

In this paper, we have described a novel MIMO-SESS technique with iterative detection as a means to provide temporal and spatial diversities for wireless communications over

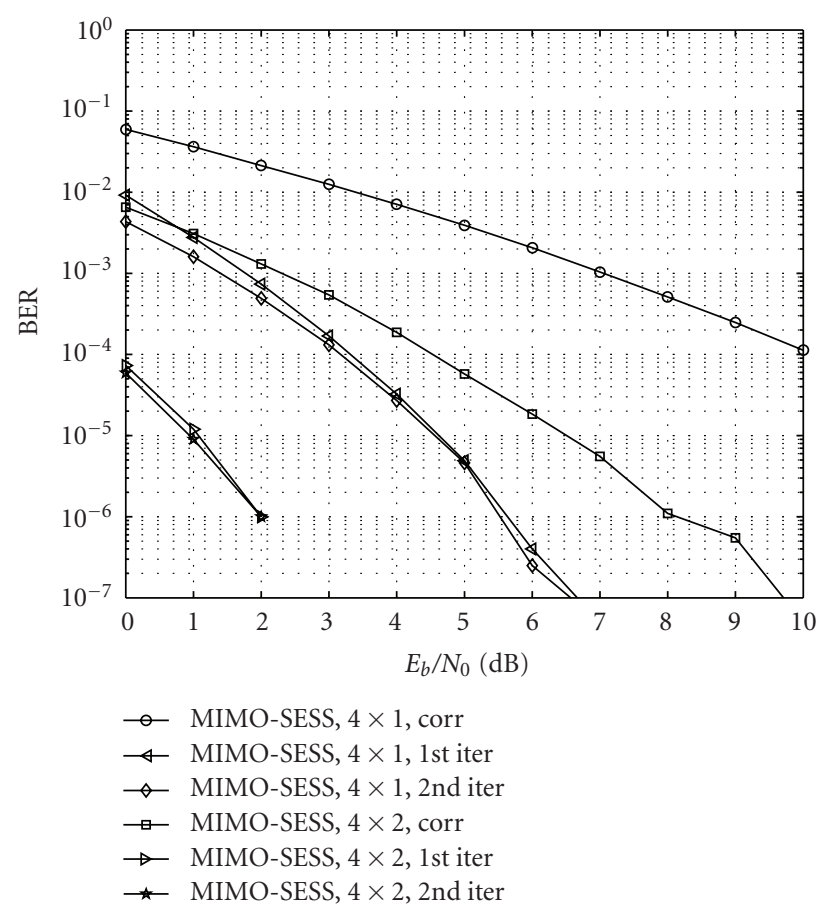

FIgure 6: Performance of the proposed MIMO-SESS system with iterative detection, four transmit antennas using half-rate code $g_{4}$, $N=64$.

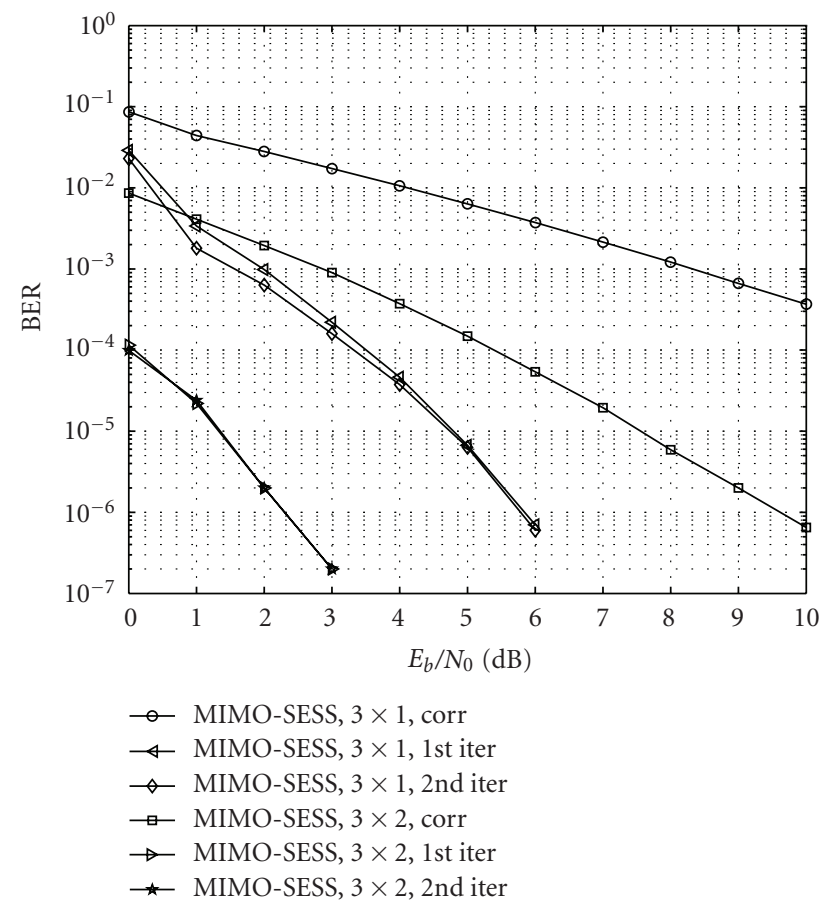

Figure 7: Performance of the proposed MIMO-SESS system with iterative detection, three transmit antennas using half-rate code $g_{3}$, $N=64$.

fading channels. The proposed scheme combines SESS with MIMO space-time block coding in a decoupling manner 


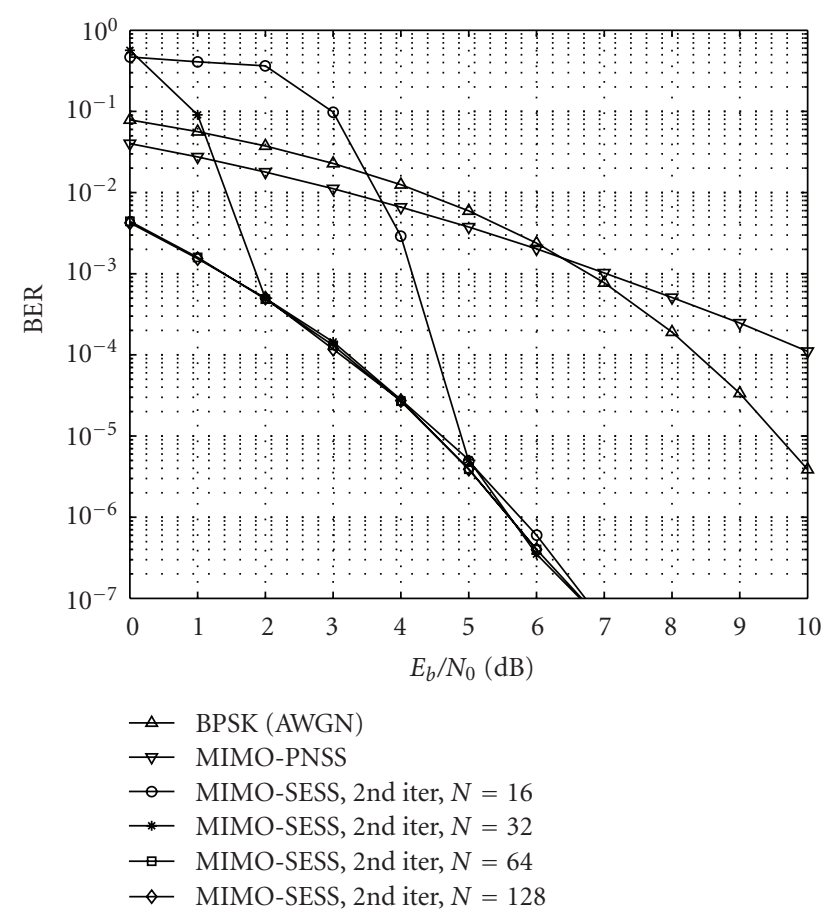

Figure 8: Performance of the proposed $2 \times 2$ MIMO-SESS system with iterative detection, $N=16,32,64,128$.

such that the number of antennas is not constrained by SESS structure. Our work has examined example OSTBC with two to four transmit antennas and one to two receive antennas in fast Rayleigh fading channels. The results show that SESS with iterative detection can significantly improve the overall BER performance, by as much as $6.7 \mathrm{~dB}$ gain over MIMOPNSS for a $2 \times 2$ antenna configuration. The linear iterative detection is based on soft estimates and has been found to converge quickly after only one iteration, making it very efficient for a practical implementation.

Our work has shown that significant diversity gain can be exploited from SESS-modulated signals such that the proposed system can completely mitigate the fading effect and achieve a BER performance of only $3.3 \mathrm{~dB}$ SNR at $10^{-4}$ BER for the $2 \times 2$, to just less than $0 \mathrm{~dB}$ for the $4 \times 2$. The performance will be poorer if the channel fading is slow and the symbols experience block fading because the temporal diversity gain with SESS will be reduced, especially if the fading block length exceeds the spreading length. In general, the diversity loss due to slow or block fading could be compensated for and recovered with interleaving.

The approach in this paper could be extended to multicode MIMO-SESS for BER improvement while enhancing the system throughput. Similarly, we anticipate that the spectral efficiency of the system can be further improved by incorporating QAM with SESS and by performing self encoding across multiple QAM symbols. We whould also like to point out that the decoupling nature of the proposed MIMO-SESS technique suggests the intriguing possibility of a coupled approach that may be advantageous. The design of such an encoding and decoding scheme (possibly together with error correction capability) for MIMO-SESS and its performance in fading channels could be an interesting and challenging problem.

\section{Acknowledgments}

The authors would like to express their appreciation for the valuable comments from the editor as well as the anonymous reviewers of their manuscript. This work was funded in part by Contract award FA9550-08-1-0393 from the U.S. Air Force Office of Scientific Research. They wish to thank Dr. J. Sjogren for his support of this study.

\section{References}

[1] L. Nguyen, "Self-encoded spread spectrum communications," in Proceedings of the IEEE Military Communications Conference (MILCOM '99), vol. 1, pp. 182-186, October 1999.

[2] L. Nguyen, "Self-encoded spread spectrum and multiple access communications," in Proceedings of the 6th IEEE International Symposium on Spread Spectrum Techniques and Applications, vol. 2, pp. 394-398, Parsippany, NJ, USA, September 2000.

[3] L. Chi, Y. H. Jung, W. M. Jang, and L. Nguyen, "Selfencoded spread spectrum with iterative detection in multi-rate multimedia communication systems," in Proceedings of the 6th International Conference on Digital Content, Multimedia Technology and its Applications (IDC '10), Seoul, South Korea, August 2010.

[4] P. Duraisamy and L. Nguyen, "Coded-sequence self-encoded spread spectrum communications," in Proceedings of the IEEE Global Telecommunications Conference (GLOBECOM '09), pp. 1-5, Honolulu, Hawaii, USA, 2009.

[5] Y. S. Kim, W. M. Jang, Y. Kong, and L. Nguyen, "Chipinterleaved self-encoded multiple access with iterative detection in fading channels," Journal of Communications and Networks, vol. 9, no. 1, pp. 50-55, 2007.

[6] A. Tehrani, R. Negi, and J. Cioffi, "Space-time coding over a code division multiple access system," in Proceedings of the IEEE Wireless Communications and Networking Conference (WCNC '99), New Orleans, La, USA, September 1999.

[7] H. Huang, H. Viswanathan, and G. J. Foschini, "Achieving high data rates in CDMA systems using BLAST techniques," in Proceedings of the IEEE Global Telecommunications Conference (GLOBECOM '99), vol. 5, pp. 2316-2320, Rio de Janeiro, Brazil, December 1999.

[8] B. Hochwald, T. L. Marzetta, and C. B. Papadias, "A transmitter diversity scheme for wideband CDMA systems based on space-time spreading," IEEE Journal on Selected Areas in Communications, vol. 19, no. 1, pp. 48-60, 2001.

[9] D. Gesbert, M. Shafi, D.-S. Shiu, P. J. Smith, and A. Naguib, "From theory to practice: an overview of MIMO space-time coded wireless systems," IEEE Journal on Selected Areas in Communications, vol. 21, no. 3, pp. 281-302, 2003.

[10] A. J. Paulraj, D. A. Gore, R. U. Nabar, and H. Bölcskei, "An overview of MIMO communications-a key to gigabit wireless," Proceedings of the IEEE, vol. 92, no. 2, pp. 198-217, 2004.

[11] A. Paulraj, R. Nabar, and D. Gore, Introduction to SpaceTime Wireless Communications, Cambridge University Press, Cambridge, UK, 2003. 
[12] S. Ma, L. Nguyen, W. M. Jang, and Y. Yang, "Performance enhancement in MIMO self-encoded spread spectrum systems by using multiple codes," in Proceedings of the 33rd IEEE Sarnoff Symposium, Princeton, NJ, USA, April 2010.

[13] J. G. Proakis, Digital Communications, McGraw-Hill, New York, NY, USA, 4th edition, 1989.

[14] B. D. Van Veen and K. M. Buckley, "Beamforming: a versatile approach to spatial filtering," IEEE ASSP Magazine, vol. 5, no. 2, pp. 4-24, 1988.

[15] D. J. Love, R. W. Heath Jr., and T. Strohmer, "Grassmannian beamforming for multiple-input multiple-output wireless systems," IEEE Transactions on Information Theory, vol. 49, no. 10, pp. 2735-2747, 2003.

[16] B. C. Lim, W. A. Krzymień, and C. Schlegel, “Transmit antenna selection for sum rate maximization in transmit zero-forcing beamforming," in Proceedings of the 10th IEEE Singapore International Conference on Communications Systems (ICCS '06), October 2006.

[17] Y. Ma, A. Leith, and R. Schober, "Predictive feedback for transmit beamforming with delayed feedback and channel estimation errors," in Proceedings of the IEEE International Conference on Communications (ICCS '08), pp. 4678-4682, Beijing, China, May 2008.

[18] V. Tarokh, H. Jafarkhani, and A. R. Calderbank, "Space-time block codes from orthogonal designs," IEEE Transactions on Information Theory, vol. 45, no. 5, pp. 1456-1467, 1999.

[19] G. Ganesan and P. Stoica, "Space-time block codes: a maximum SNR approach," IEEE Transactions on Information Theory, vol. 47, no. 4, pp. 1650-1656, 2001.

[20] W. Su, X.-G. Xia, and K. J. R. Liu, "A systematic design of high-rate complex orthogonal space-time block codes," IEEE Communications Letters, vol. 8, no. 6, pp. 380-382, 2004.

[21] G. J. Foschini, "Layered space-time architecture for wireless communication in a fading environment when using multielement antennas," Bell Labs Technical Journal, vol. 1, no. 2, pp. 41-59, 1996.

[22] D. J. Love and R. W. Heath Jr., "Limited feedback precoding for spatial multiplexing systems," in Proceedings of the IEEE Global Communications Conference (GLOBECOM '03), vol. 4, pp. 15, San Francisco, Calif, USA, December 2003.

[23] A. S. Khrwat, B. S. Sharif, C. C. Tsimenidis, and S. Boussakta, "Channel prediction for precoded spatial multiplexing multiple-input multiple-output systems in time-varying fading channels," IET Signal Processing, vol. 3, no. 6, pp. 459-466, 2009.

[24] V. Tarokh, H. Jafarkhani, and A. R. Calderbank, "Spacetime block codes for wireless communications: performance results," IEEE Transaction on Information Theory, vol. 45, no. 5, pp. 1456-1467, 1999.

[25] S. M. Alamouti, "A simple transmit diversity technique for wireless communications," IEEE Journal on Selected Areas in Communications, vol. 16, no. 8, pp. 1451-1458, 1998.

[26] K. Hua, L. Nguyen, and W. M. Jang, "Synchronisation of selfencoded spread spectrum system," Electronics Letters, vol. 44, no. 12, pp. 749-751, 2008. 

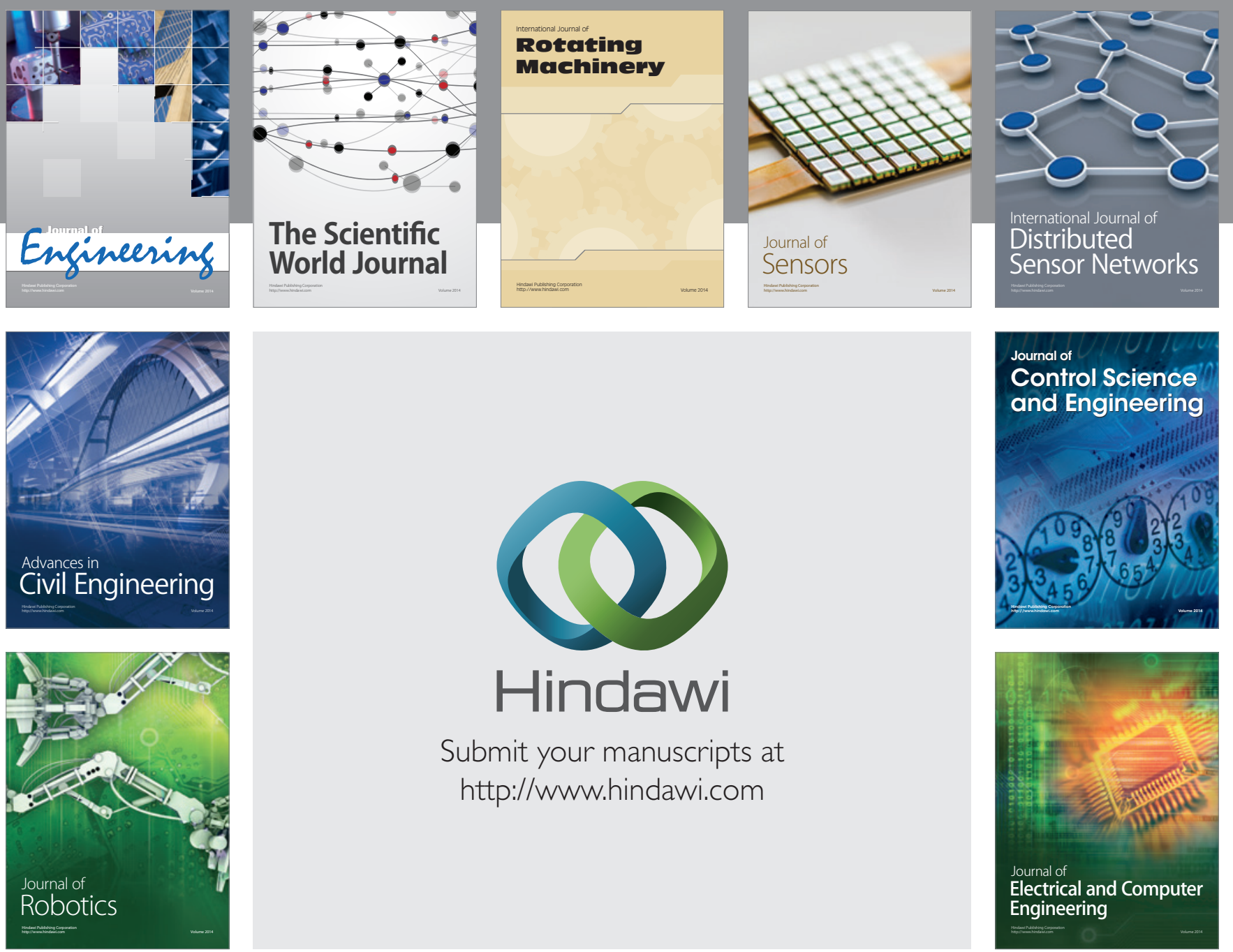

Submit your manuscripts at

http://www.hindawi.com
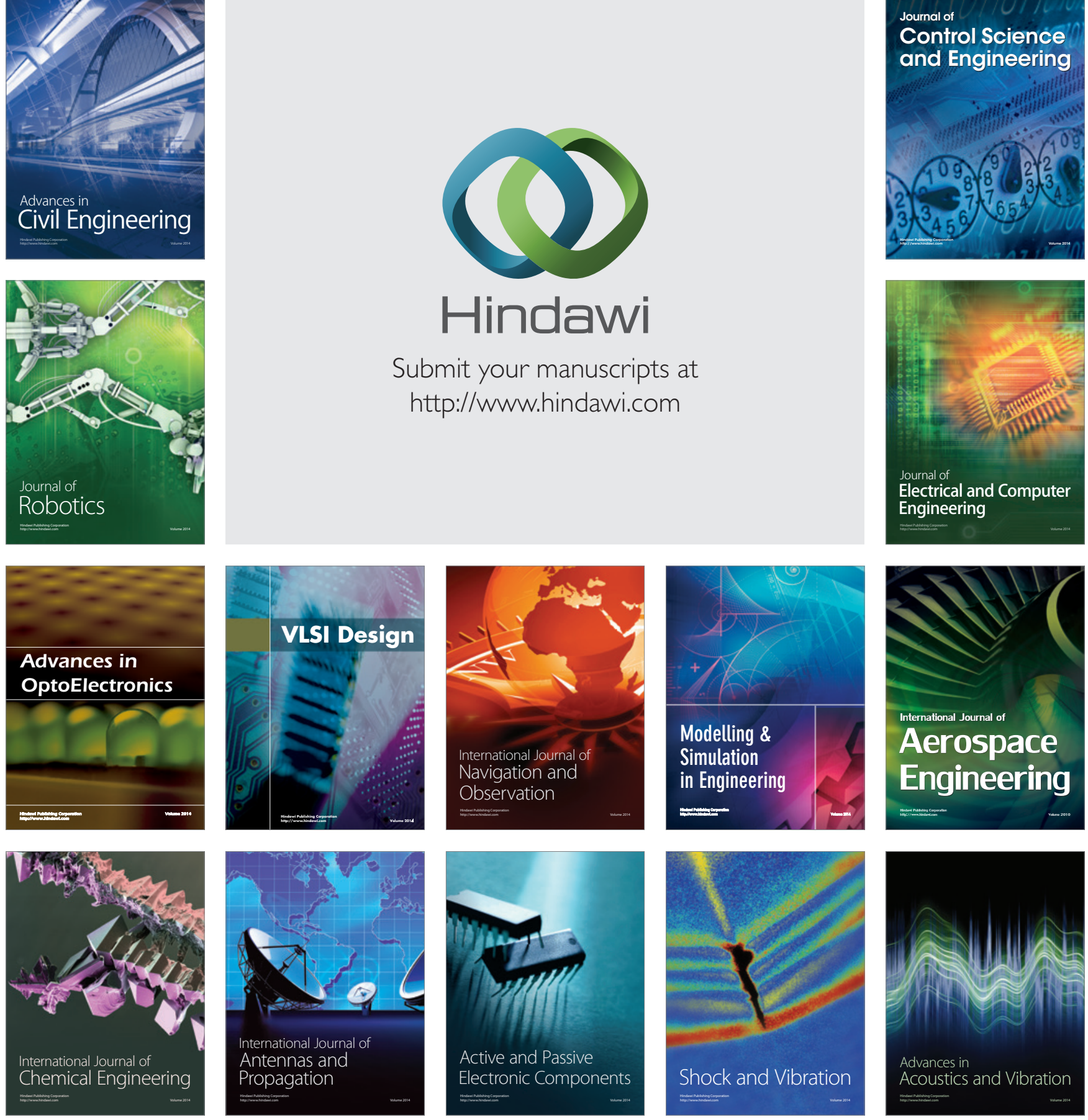